\title{
Age-related changes in processing speed: unique contributions of cerebellar and prefrontal cortex
}

\author{
Mark A. Eckert ${ }^{1}$, Noam I. Keren', Donna R. Roberts ${ }^{2}$, Vince D. Calhoun ${ }^{3}$ and Kelly C. Harris ${ }^{1}$ \\ 1 Department of Otolaryngology - Head and Neck Surgery, Medical University of South Carolina, Charleston, SC, USA \\ 2 Department of Radiology and Radiological Science, Medical University of South Carolina, Charleston, SC, USA \\ ${ }_{3}^{3}$ Department of Electrical and Computer Engineering, University of New Mexico, and the Mind Research Network, Albuquerque, NM, USA
}

\section{Edited by:}

Anna C. Nobre, University of Oxford,

UK

\section{Reviewed by:}

Jeanne Townsend, University of California San Diego, USA

A. Morcom, University of Edinburgh, UK

\section{${ }^{*}$ Correspondence:}

Mark A. Eckert, Hearing Research Program, Department of

Otolaryngology - Head and Neck

Surgery, 135 Rutledge Avenue, MSC

550, Charleston, SC 29425-5500, USA.

e-mail:eckert@musc.edu
Age-related declines in processing speed are hypothesized to underlie the widespread changes in cognition experienced by older adults. We used a structural covariance approach to identify putative neural networks that underlie age-related structural changes associated with processing speed for 42 adults ranging in age from 19 to 79 years. To characterize a potential mechanism by which age-related gray matter changes lead to slower processing speed, we examined the extent to which cerebral small vessel disease influenced the association between age-related gray matter changes and processing speed. A frontal pattern of gray matter and white matter variation that was related to cerebral small vessel disease, as well as a cerebellar pattern of gray matter and white matter variation were uniquely related to age-related declines in processing speed. These results demonstrate that at least two distinct factors affect age-related changes in processing speed, which might be slowed by mitigating cerebral small vessel disease and factors affecting declines in cerebellar morphology.

Keywords: aging, processing speed, cerebellum, cerebral small vessel disease, structural covariance

\section{INTRODUCTION}

A hallmark of cognitive aging is slowed processing speed, or a decrease in the rate at which people perform perceptual, motor, and decision making tasks. Because processing speed is one of the strongest predictors of performance across cognitive tasks in older adults (Salthouse and Ferrer-Caja, 2003), it is the foundation of a major hypothesis for age-related cognitive decline (Salthouse, 1996). Our understanding of age-related neural changes affecting processing speed is limited, however. This is largely because processing speed is not a unitary construct and does not involve a single neural system, but is rather a reflection of coordinated activity across multiple neural networks. Even the simplest processing speed tasks require stimulus perception, decision making and planning, motor performance, and performance evaluation. Age-related changes in some or all of the networks that support these functions could affect behavioral measures of processing speed. For this reason a systems approach may be essential for understanding the neurobiology of age-related declines in processing speed.

Early neurobiological explanations for age-related changes in processing speed focused on cortical declines in neuropil and myelin (Morris and McManus, 1991; Salthouse, 1992; Miller, 1994). More recently, whole brain gray matter volume and white matter fractional anisotropy have been associated with processing speed (Coffey et al., 2001; Charlton et al., 2006; Vernooij et al., 2008; Schiavone et al., 2009). These results appear to be due, in part, to regionally specific structural declines in attention-related neural systems, particularly within prefrontal cortex (Kennedy and Raz, 2009; Bartzokis et al., in press). Additional structural declines in motor and sensory cortex are likely to contribute to age-related changes in processing speed, however, given the important contribution of perceptual and motor function to processing speed measures (Welford, 1981; Salthouse, 2000).

One pathophysiological mechanism underlying the structural changes associated with processing speed appears to be cerebral small vessel disease (Gunning-Dixon and Raz, 2000; Prins et al., 2005; Au et al., 2006; van den Heuvel et al., 2006; van Dijk et al., 2008). While the widespread pattern of cerebral small vessel disease likely affects a variety of neural systems, there is evidence that it reduces engagement of dorsolaterateral prefrontal cortex during working memory tasks (Nordahl et al., 2006) and slows processing speed (Kochunov et al., 2010). It is not clear, however, whether these frontal effects account for all the age-related anatomical changes underlie processing speed or whether there are additional networks that contribute to age-related declines in processing speed.

This cross-sectional study was designed to identify putative neural networks exhibiting age-related structural changes that account for age-related changes in processing speed. Structural networks appear to share topological features with functional networks (He et al., 2007; Honey et al., 2007), due in part to underlying long and short range fiber connections between regions (Bullmore and Sporns, 2009). As a consequence, structural and functional networks can be affected in conditions such as Alzheimer's disease and multiple sclerosis (He et al., 2008, 2009). We examined the structural covariance of voxels across the brain to test the hypothesis that distinct patterns of gray matter covariance would predict age-related changes in processing speed, compared to a single prefrontal network. We also examined the extent to which cerebral small vessel disease could explain the link 
between age-related changes in structural networks and processing speed, thus providing evidence of a potential mechanism for age-related structural changes that have a broad impact on cognitive function.

\section{MATERIALS AND METHODS PARTICIPANTS}

Forty-two healthy adults (age range 19-79; 64\% female; mean education of 18.2 years; mean Hollingshead socioeconomic status of 51.8) were recruited from the Medical University of South Carolina (MUSC) community and the Charleston, S.C. area. To be included in this study, subjects had to score a 27 or higher on the Mini-Mental Status Exam, have no history of neurologic or psychiatric events, be free of central nervous system acting medications, and meet the safety criteria for MRI scanning. The older adults ( $>60$ years) in this study did not report a history of significant cardiovascular problems (e.g., heart attack, stent placement, or bypass surgery). In addition, there was no evidence that taking anti-hypertension medications in the older adults ( 8 of 18 subjects) was related to the structural or behavioral measures described below. Informed consent was obtained from each participant for this Medical University of South Carolina Institutional Review Board-approved project and the experiments were conducted in accordance to the Declaration of Helsinki.

\section{PROCESSING SPEED ASSESSMENT}

A large number of cognitive processes are involved in processing speed tasks. Processing speed was operationally defined in this study as (1) perceptual and motor processing speed using tasks that place limited demands on working memory and inhibition of responses, as well as (2) executive processing speed that was assessed by requiring subjects to alternate between behavioral plans. The executive processing speed task required subjects to continuously inhibit the rule guiding the previous behavior and maintain stimulus representations in working memory.

The two processing speed constructs were measured using the Connections Test (Salthouse et al., 2000), a variant of the Trail Making Test (Reitan, 1992), in which subjects connect circled letters and/or numbers in alphabetic and numeric sequence. The letters and/or numbers are pseudo-randomly organized in a $7 \times 7$ array of circles on a sheet of paper, with the next target located at an adjacent location. The close proximity of the adjacent target is designed to limit the motor demands of the task (Salthouse et al., 2000). In one form of the test, Connections Simple, subjects connect as many numbers or letters as possible in $20 \mathrm{~s}$. Subjects complete two trials of the letters form and two trials of the numbers form. Performance is averaged across the four trials to provide a single estimate of processing speed. This test is postulated to involve target identification, search and perceptual comparison to verify target, response, and determination of the next item in the sequence. For the other form of the test, Connections Complex, subjects alternate between drawing a line between a number and a letter, again in alphabetic and numeric sequence. Subjects complete two trials starting with a number and two trials starting with a letter. Performance is averaged across the four trials to provide a single estimate of processing speed. In addition to the perceptual and motor processes required for identifying the well entrained symbols, the Connections Complex test requires subjects to switch between stimulus types (letters and numbers) thereby engaging working memory and inhibitory systems. By removing Connections Simple performance from the Connections Complex score, the Connections Test provides estimates of executive processing speed (Connections Complex), as well as perceptual and motor processing speed (Connections Simple). The Connections Test was selected for this study because performance is robustly affected by age, relates strongly to other measures of processing speed, and predicts age-related cognitive changes for constructs such as reasoning (Salthouse, 2005), thereby providing generalization of findings from this study to other indices of processing speed and cognition.

Sub-tests from the Woodcock-Johnson Tests of Achievement and Cognitive Abilities (Woodcock et al., 2001) were administered to determine the extent to which age-related changes were specific to processing speed or observed across cognitive measures. The subtests included a test of working memory in which subjects repeat in reverse order a list of numbers orally presented to them (Numbers Reversed), a test of vocabulary knowledge in which subjects provide the names for drawings of objects (Picture Vocabulary), and a processing speed test in which subjects circle identical numbers in a row of six numbers (Visual Matching). In the Visual Matching test, task difficulty increases as the number of digits in the stimuli increases from one digit numbers to three digit numbers. The number of correct items completed in $3 \mathrm{~min}$ is used as the estimate for processing speed. The Visual Matching test is similar to the Connections Simple test in that it places demands on perceptual and motor speed. For this reason, the Visual Matching test provided a measure to verify results from the Connections Simple test. The Numbers Reversed test was used to verify that Connections Complex performance, after controlling for Connections Simple, was related to working memory. Numbers Reversed data was missing for two subjects. The Picture Vocabulary test is un-timed and was used to examine the specificity of anatomical associations with processing speed compared to vocabulary knowledge, a cognitive domain that is relatively preserved with increasing age (Singer et al., 2003).

The raw scores of the Connections and Woodcock-Johnson sub-tests were converted to within-sample $Z$-scores, based on the mean and standard deviation of the scores for each test, so that the behavioral tests and structural covariance measures described below had the same psychometric properties. The raw scores for all of the behavioral measures are presented in Table 1 , along with the cognitive construct that each measure is thought to assess.

\section{IMAGE ACQUISITION}

Structural images were collected on a Philips 3T Intera scanner (Philips Medical Systems, The Netherlands) using an eight-channel SENSE head coil (reduction factor $=2$ ). T1-weighted images were collected for brain structure analyses (160 slices with a $256 \times 256$ matrix, $\mathrm{TR}=8.13 \mathrm{~ms}, \mathrm{TE}=3.70 \mathrm{~ms}$, flip angle $=8^{\circ}$, slice thickness $=1.0 \mathrm{~mm}$, and no slice gap). Fluid-attenuated inversion-recovery (FLAIR) images were collected to identify regions of cerebral small vessel disease ( 60 slices with a $256 \times 256$ matrix, TR $=1100 \mathrm{~ms}$, $\mathrm{TE}=125 \mathrm{~ms}$, flip angle $=90^{\circ}$, slice thickness $=2.00 \mathrm{~mm}$, and no slice gap). 
Table 1 | Mean and SD of the raw scores from the behavioral measures and the cognitive domain that each measures is proposed to assess.

\begin{tabular}{|c|c|c|c|}
\hline Test name & Mean raw score (SD) & Cognitive domain construct & Source \\
\hline Connections Simple ${ }^{\dagger}$ & $27.08(8.58)$ & Perceptual and motor processing speed & Salthouse et al. (2000) \\
\hline Connections Complex ${ }^{\dagger}$ & $15.14(5.58)$ & $\begin{array}{l}\text { Executive processing speed (perceptual } \\
\text { and motor + working memory, task } \\
\text { switching, and response inhibition) }\end{array}$ & Salthouse et al. (2000) \\
\hline Visual Matching $^{\dagger}$ & $50.86(7.82)$ & Perceptual and motor processing speed & WJ III -Tests of Cognitive Abilities \\
\hline
\end{tabular}

${ }^{\dagger} N=42$.

${ }^{\ddagger} N=40$.

\section{STRUCTURAL IMAGE PROCESSING}

A standardized template composed of young subjects (e.g., ICBM-152) is often used in imaging studies to normalize individual images into a common coordinate system for comparison. To ensure that images from younger and older subjects were properly co-registered to a common coordinate space, a study-specific template was created using unified segmentation and diffeomorphic image registration (DARTEL) in SPM5 (Ashburner and Friston, 2005; Ashburner, 2007). Unified segmentation was performed simultaneously to bias-field correct and segment each subject's image into native space gray matter, white matter, and CSF probabilistic images. The segmented gray matter images were then normalized using the DARTEL procedure, which involves diffeomorphic registration to preserve cortical topology using a membrane bending energy or Laplacian model (Ashburner, 2007). This procedure creates invertible and smooth deformations of each subject's native space gray matter image into a common coordinate space, as well as producing a template that is representative of the brain size and shape of all the participants (Harris et al., 2009). The DARTEL procedure and creation of a study-specific template were chosen for this aging study to reduce normalization error that might occur with other normalization procedures. An analysis of the Jacobian determinant images, obtained from the gray matter DARTEL flow fields, demonstrated age-related changes in the extent to which images from the youngest and oldest subjects had to be displaced to fit to the template (Figure 1). However, the DARTEL procedure provided for consistent spatial registration of brain regions across the sample (Figure 1). Therefore, modulation was performed to adjust the local signal intensity of gray matter or white matter voxels to reflect the degree of volumetric displacement that occurred during normalization. Finally, a Gaussian smoothing kernel of $8 \mathrm{~mm}$ was used to ensure that the data were normally distributed and to limit false positive results ${ }^{1}$.

\section{SOURCE BASED MORPHOMETRY}

Source based morphometry (SBM) is a multivariate analysis that, like voxel based morphometry (VBM), can be used to examine the relation between regional gray matter volume or white matter volume and a dependent variable such as processing speed (Xu et al., 2009a,b). Unlike VBM, SBM decomposes variation in gray matter and white matter across the images of subjects into

${ }^{1}$ www.fil.ion.ucl.ac.uk/ john/misc/dartel_guide.pdf sources of common variance, thus effectively reducing the problem of multiple comparisons that is present in VBM analyses in which thousands of voxels are examined in multiple univariate comparisons. Because specific patterns of gray matter covariance have been related spatially to patterns of activation in functional networks (Honey et al., 2007; Bullmore and Sporns, 2009), SBM provides the opportunity to determine the extent to which variation in gray matter across the source or component predicts processing speed. Specific examples of structural components that are spatially consistent with functional networks, such as the default mode network, are presented below.

In addition to identifying variation in anatomy that may underlie functional networks, SBM also provides the opportunity to: (1) identify the extent to which a component is driven by a common factor that would cause brain regions to scale together, such as brain size; and (2) identify an etiological factor such as cerebral small vessel disease that could affect distinct cortical regions due to the deafferentation stemming from white matter pathology. In sum, SBM was used in this study to identify distinct patterns of age-related change in brain morphology.

SBM was performed using the GIFT toolbox version $1.3 \mathrm{~g}^{2}$. The normalized, modulated, and smoothed gray matter and white matter images from all 42 subjects were first analyzed to estimate the total number of components by sub-sampling the images until the estimated entropy rate was equivalent to the entropy rate of an independent random process with a Gaussian distribution. To determine the number of components, dimension estimation was performed of components, was performed using the minimum description length (MDL) criteria and modified to account for spatial correlation (Li et al., 2007). The MDL criteria estimates the number of components from the maximum log-likelihood of the images (number of sub-sampled voxel by sample size and the log of the covariance matrix across the sample), with a penalty for complexity based on the number of free parameters (sample size). This analysis yielded an estimate of seven gray matter and four white matter independent components.

Independent component analysis was then performed using an infomax algorithm that minimizes the mutual information of neural networks (Bell and Sejnowski, 1995). Each of the 42 images was converted into a single vector, thereby creating a 42 -case row by gray matter voxel or white matter voxel matrix. This matrix was decomposed

${ }^{2}$ http://icatb.sourceforge.net 


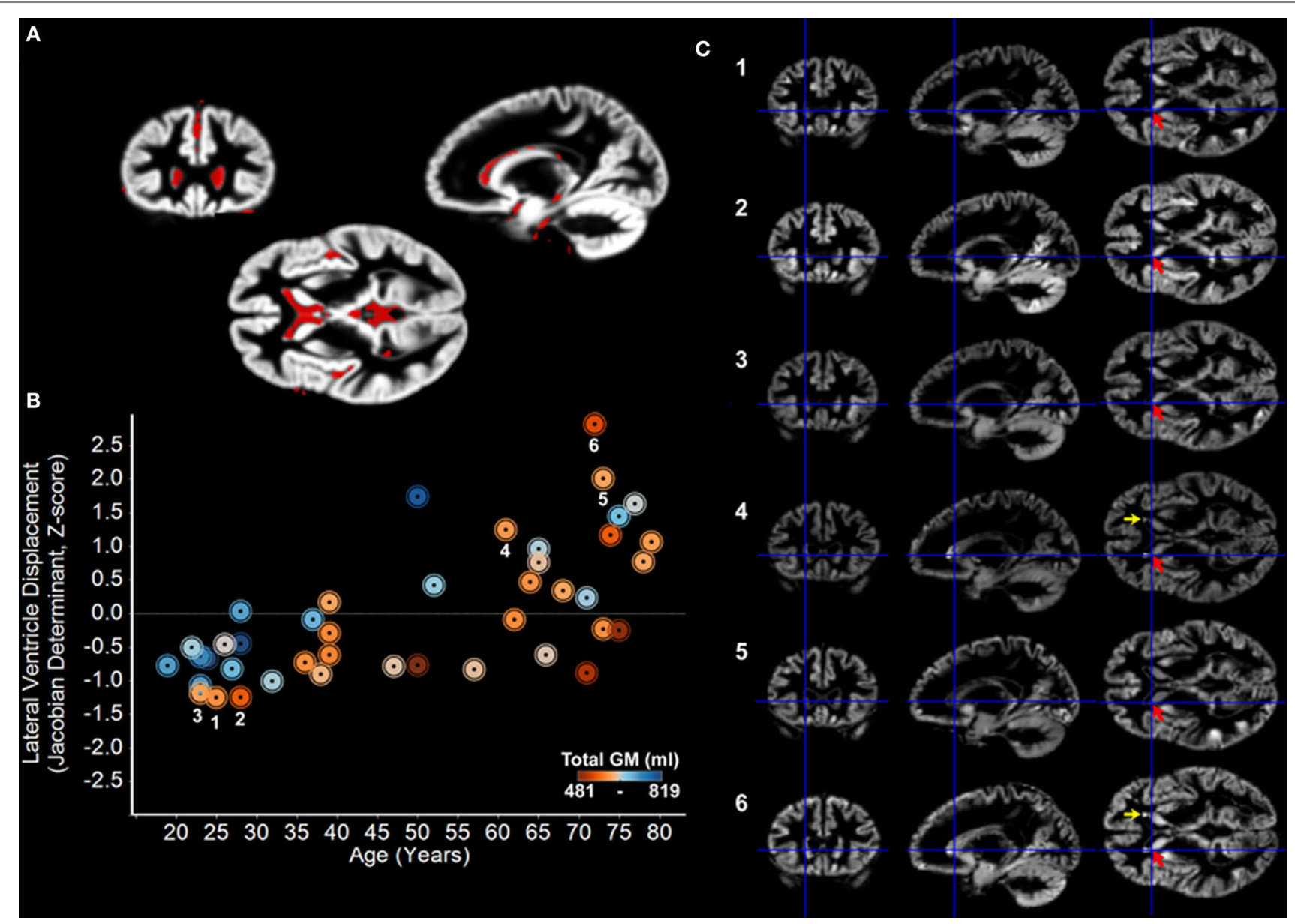

FIGURE 1 | Images from the youngest and oldest adults required the most volume displacement during normalization, but visual inspection demonstrates that there were no DARTEL normalization errors due to this displacement. (A) Jacobian determinant images, that reflect the degree of volumetric displacement during normalization, were obtained from the DARTEL gray matter flow fields. There was significant age-related variation in the extent to which regions surrounding the ventricles and CSF in general required displacement during normalization ( $1 \% \mathrm{FDR}$ ). The results are overlaid on the study-specific DARTEL template. (B) An estimate leigenvariate from the age-related effect shown in (A)] of how much each subject's lateral ventricles had to be displaced to fit to the template is plotted across age demonstrating that many younger, middle-aged, and older subjects required little ventricle displacement. Note that total gray matter volume was not related to the amount of ventricular displacement (GM, gray matter). (C) Visual inspection of the normalized gray matter images in three young female subjects and three of the oldest female subjects [labeled numerically in (B)] requiring the most volume displacement demonstrates that all were appropriately normalized to a common space. Note the close correspondence in the position of the head of the caudate across the six subjects (red arrows). Note also that there were areas where white matter regions were segmented as gray matter in the older adults (yellow arrows) and identified using SBM (Figure 2). into a source matrix that represents the degree to which each voxel contributes to a component, as well as a mixing matrix that represents a standardized estimate of tissue volume within each independent component for each subject. In other words, people with low gray matter or white matter values within one brain region of the component are likely to have low gray matter or white matter values, respectively, in another region within the component. Therefore, SBM is analogous to the volumetric comparison performed in VBM analyses, while examining data that has been reduced into gray matter or white matter constructs representing the values from across the source.

\section{WHITE MATTER HYPERINTENSITY MEASURES}

Guided by previous studies in which small cerebral vessel disease was measured on MRI scans (Prins et al., 2005; van Dijk et al., 2008), the number of distinct WMH (white matter hyperintensi- ties) was counted in each subject's FLAIR image. A series of image processing steps were taken to increase the reliability of WMH classification. The FLAIR images were normalized into the gray matter DARTEL space by co-registering the original FLAIR and T1 scans using the SPM5 mutual information function and then applying the DARTEL normalization parameters to the FLAIR images. WMH were defined as clusters of at least two adjacent voxels across two image sections that had at least a 30\% increase in signal intensity relative to the mean of the white matter signal (Trebbe et al., 2009) within the 50\% probability DARTEL white matter template. The intra- and inter-rater reliability for the volume of labeled WMH was Cronbach's $\alpha=0.93$ and 0.90, respectively. The frequency of WMH across subjects did not have a normal distribution (Kolmogorov-Smirnov test of normal distribution: $Z=1.90, p<0.001)$. For this reason, cerebral small vessel 
disease was categorized as: (1) not present; (2) mild; or (3) modest to pronounced, based on the distribution of WMH among people with WMH. This classification was compared with a neuroradiologist's (author Donna R. Roberts) clinical assessment of the scans where WMH were considered clinically typical for the demographic characteristics of the subjects and were classified as: (1) not present; (2) scattered non-specific white matter foci; and (3) scattered non-specific white matter foci as well as periventricular white matter foci. The reliability between the WMH counts and radiological assessment was Cronbach's $\alpha=0.89$. This association validates the WMH count classification, particularly given that the radiological assessment was performed with demographic information. There was no evidence of major vessel infarcts and all subjects were typically functioning adults with no reported history of cerebral vascular events.

To create a density map describing the spatial location of WMH across subjects, $\mathrm{WMH}$ regions exhibiting a $30 \%$ increase in signal relative to the mean white matter signal were manually labeled on the normalized FLAIR images and a binary image was created representing WMH regions for each subject. These images also were used to perform non-parametric lesion-symptom mapping using the NPM software ${ }^{3}$ (Rorden et al., 2007), in which processing speed was related to the presence or absence of WMH at each voxel location in the brain (1\% FDR). This analysis highlighted brain regions where $\mathrm{WMH}$ were likely to occur and be associated with slow processing speed.

\section{STATISTICS}

Multiple regression and correlation analyses were performed to characterize the extent to which age was associated with the cognitive measures, as well as the gray matter components. We hypothesized that multiple neural systems would uniquely predict age-related changes in processing speed. To test this hypothesis, Pearson correlation and multiple regression were performed to examine the extent to which a single gray matter component or multiple components were associated with age-related changes in processing speed. We then examined the extent to which the white matter components would (1) predict additive variance in processing speed or (2) were related to the gray matter components and provided evidence of a specific neural system that was affected by age and related to processing speed. To test the prediction that cerebral small vessel disease underlies structural changes associated with age-related changes in processing speed, multiple regression was subsequently performed to examine the extent to which WMH predicted processing speed and the gray matter components.

\section{RESULTS}

\section{AGE-RELATED CHANGES IN COGNITION}

Table 2 demonstrates that processing speed slowed with increasing age. After controlling for Connections Simple performance, however, age was no longer significantly associated with Connections Complex or Numbers Reversed and the strength of relation with Visual Matching was diminished. Numbers Reversed was positively related to Connections Complex performance after covarying out Connections Simple performance $\left[r_{(39)}=0.34, p<0.05\right]$, thereby

${ }^{3}$ http://www.sph.sc.edu/comd/rorden/npm/
Table 2 | Pearson correlations between age and the behavioral measures.

\begin{tabular}{llllll}
\hline $\begin{array}{l}\text { Connections } \\
\text { Simple }\end{array}$ & $\begin{array}{l}\text { Connections } \\
\text { Complex }\end{array}$ & $\begin{array}{l}\text { Visual } \\
\text { Matching }\end{array}$ & $\begin{array}{l}\text { Numbers } \\
\text { Reversed }\end{array}$ & $\begin{array}{l}\text { Picture } \\
\text { Vocabulary }\end{array}$ \\
\hline Age & $-0.72^{* * *}$ & $-0.66^{* * *}$ & $-0.75^{* * *}$ & $-0.32^{*}$ & -0.08 \\
Age $^{\dagger}$ & - & -0.13 & $-0.31^{*}$ & -0.06 & 0.10 \\
\hline
\end{tabular}

${ }^{*} p<0.05, * * * p<0.001$

${ }^{+}$Connections Simple Covaried.

supporting the premise that Connections Complex is uniquely measuring executive function such as working memory compared to Connections Simple. These results indicate that processing speed, particularly involving perceptual and motor function, exhibited cross-sectional decline with age.

\section{GRAY MATTER COMPONENTS AND AGE-RELATED CHANGES}

Figure 2 presents the seven independent gray matter components that were identified using SBM. They included: (1) a component involving ventromedial prefrontal cortex, posterior cingulate and bilateral parietal regions that have a spatial pattern that is similar to the default mode network; (2) a bilateral temporal pole, posterior cerebellar hemisphere, motor, prefrontal, and anterior and midcingulate component; (3) a brainstem, right superior frontal sulcus, right posterior cingulate sulcus, left inferior temporal gyrus, left inferior frontal gyrus, and left cerebellar hemisphere component; (4) a bilateral superior cerebellar and intraparietal sulcus component; (5) a bilateral visual cortex component; (6) a bilateral anterior insula, basal ganglia, anterior medial temporal lobe, inferior and lateral cerebellar, and occipital-temporal-parietal junction (MT) component and (7) an inferior temporal gyrus and inferior cerebellar component.

Age was significantly related to gray matter volume variation within components $1,3,4,6$, and 7 . The relation between age and component 1 (default mode network), in particular, was explained by the strong relation between total gray matter volume and component $1\left[r_{(41)}=0.90, p<0.001\right]$. Table 3 shows that the association between age and components 4,6 , and 7 were largely independent of global changes in gray matter volume.

In contrast to the other components, component 7 exhibited an age-related increase in tissue volume, due in part to classification of white matter as gray matter in areas anterior to the horn of the lateral ventricles (orange arrows in Figure 2, component 7 ) where there are WMH (Figure 4A). The inverse of component 7 ( $7 a$ in Figure 1) includes regions where there is less gray matter relative to the regions in component 7 after controlling for variance attributable to the other gray matter components. These regions exhibit age-related decreases in volume, including anterior cingulate cortex (ACC) and dorsolateral prefrontal cortex regions in component $7 a$ [e.g. ACC region with age: $r_{(41)}=-0.37, p<0.05$ ].

Table 3 demonstrates that gray matter components 2, 3, 4, 7 predicted processing speed $(p<.01$ to $p<.001)$. These four components were entered in the first level of a hierarchical multiple regression analysis that demonstrated component 2 (std. beta $=0.24$, $p<0.05)$, component 4 (std. beta $=0.36, p<0.01$ ), and component 7 (std. beta $=-0.41, p<0.001)$ predicted significant unique variance 


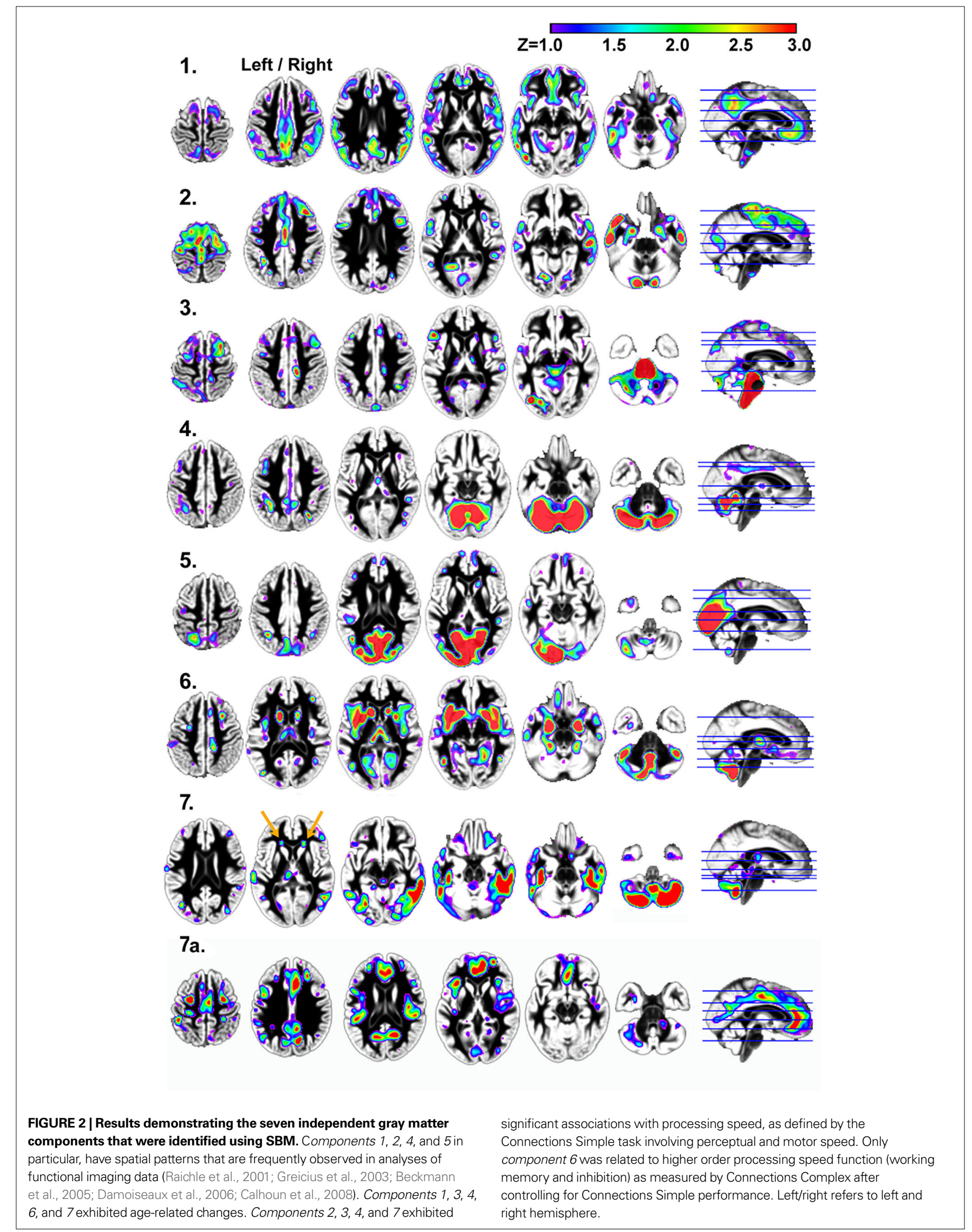


Table 3 |The Pearson correlation of each gray matter component with each behavioral measure is presented to show the specificity of the components to each processing speed measure, before and after controlling for perceptual and motor speed that was measured by Connections

Simple. Note the consistently strong correlations for components 4 and 7 with measures of perceptual and motor processing speed.

\begin{tabular}{|c|c|c|c|c|c|c|c|}
\hline & Component 1 & Component 2 & Component 3 & Component 4 & Component 5 & Component 6 & Component 7 \\
\hline Age & $-0.34^{*}$ & -0.21 & $0.42^{* *}$ & $-0.61 * * *$ & -0.10 & $-0.46^{* *}$ & $0.53^{* * *}$ \\
\hline Age (total GM covaried) & 0.17 & -0.06 & 0.23 & $-0.52 * * *$ & 0.13 & $-0.48 * * *$ & $0.46^{* *}$ \\
\hline Connections Simple & 0.26 & $0.42 * *$ & $-0.42 * *$ & $0.50 * * *$ & 0.02 & 0.26 & $-0.52 * * *$ \\
\hline Connections Complex & 0.21 & 0.24 & $-0.43^{* *}$ & $0.43 * *$ & 0.03 & $0.43^{* *}$ & $-0.33^{*}$ \\
\hline $\begin{array}{l}\text { Connections Complex } \\
\text { (simple performance } \\
\text { removed) }\end{array}$ & -0.01 & -0.19 & -0.14 & 0.03 & 0.02 & $0.38^{*}$ & 0.18 \\
\hline Visual Matching & 0.18 & $0.33^{*}$ & $-0.42^{*}$ & $0.49 * * *$ & 0.08 & $0.42^{* *}$ & $-0.34^{*}$ \\
\hline $\begin{array}{l}\text { Visual Matching } \\
\text { (simple performance } \\
\text { removed) }\end{array}$ & -0.04 & -0.00 & -0.15 & 0.16 & 0.10 & $0.34 *$ & 0.10 \\
\hline Numbers Reversed & 0.05 & 0.05 & -0.13 & 0.09 & 0.06 & $0.36^{*}$ & -0.08 \\
\hline Picture Vocabulary & $0.19 *$ & -0.09 & -0.12 & 0.18 & $0.29 *$ & 0.03 & 0.06 \\
\hline
\end{tabular}

${ }^{*} p<0.05,{ }^{*} p<0.01,{ }^{*}{ }^{*} p<0.001$.

in Connections Simple performance (multiple $R=0.74, p<0.001$ ). However, brainstem component 3 (std. beta $=-0.12$, ns) predicted Connections Simple performance because of shared variance with the motor, prefrontal, posterior cerebellar component 2 and the superior cerebellar component 4. Figure 3 presents scatter plots of the combined predictive value of the three gray matter components for Connections Simple processing speed, as well as the unique contribution of each variable. Figure 3 demonstrates that the association with Connections Simple is particularly robust across subjects for components 4 and 7 . Age was entered in a second level of the regression and demonstrated that the unique relations between processing speed and components 4 and 7 was dependent on age (component 4: std. beta $=0.12$, ns; component 7 : std. beta $=-0.20, \mathrm{~ns})$. Age-related components 1 and 6 did not significantly predict Connections Simple performance, thereby indicating spatial specificity for age-related structural changes that predict processing speed.

Table 3 demonstrates components 4 and 7 were not related to Connections Complex after controlling for perceptual and motor variance as measured by Connections Simple. Only the inferior frontal, anterior insula, basal ganglia, amygdala, and inferior cerebellar component 6 was related to Connections Complex after controlling for Connections Simple $\left[r_{(41)}=0.38, p<0.05\right]$, thereby providing a double dissociation between components related to perceptual and motor processing speed (components 2, 4, and 7 with Connections Simple) compared to one component specifically related to executive function processing speed (component 6 with Connections Complex after controlling for Connections Simple). The strength of the relation between component 6 and Connections Complex (executive processing speed) was not affected after controlling for total gray matter volume and age [partial $r_{(39)}=0.34, p<0.05$ ], but was due in part to a common underlying association with working memory performance as measured by Numbers Reversed [partial $r_{(39)}=0.29$, $p=0.07]$.

\section{LINKAGE ACROSS WHITE MATTER AND GRAY MATTER COMPONENTS}

Figure 4 presents the four white matter components that were identified using SBM. Only white matter component 2 was significantly related to Connections Simple processing speed $\left[r_{(41)}=0.44\right.$, $p<0.01]$. This component was spatially consistent with cerebellar gray matter component 4 and included regions within the cerebellar peduncles, brainstem, and posterior limb of the internal capsule. Figure 5 shows that individual variation in component 2 white matter volume was strongly related to cerebellar component 4 gray matter volume $\left[r_{(41)}=0.68, p<0.001\right]$ and predicted the same variance in Connections Simple performance as the gray matter component 4 [white matter component 2: partial $r_{(39)}=0.16$, ns, controlling for gray matter component 4]. Spatially consistent variation in gray matter and white matter components was also observed for white matter component 4 , which included prefrontal white matter, and frontal gray matter component 7 . White matter and gray matter volume variation in these components were also significantly correlated $\left[r_{(41)}=-0.51, p<0.001\right]$.

\section{CEREBRAL SMALL VESSEL DISEASE: DECLINES IN MORPHOLOGY AND PROCESSING SPEED}

There was an age-related increase in the frequency of WMH $\left[F_{(2,41)}=16.65, p<0.001\right]$. The seventeen subjects not exhibiting WMH were significantly younger than the 16 subjects exhibiting a mild degree of $\mathrm{WMH}$ (mean difference $=17.84$ years) and the nine subjects exhibiting moderate to pronounced WMH (mean difference $=34.88$ years). Figure 6A presents a probability map demonstrating that WMH were most likely to be observed in periventricular white matter regions.

Subjects without evidence of WMH exhibited faster processing speed than subjects with mild WMH and with moderate to pronounced WMH when processing speed was measured by Connections Simple $\left[F_{(2,41)}=10.75, p<0.001\right]$, Visual Matching $\left[F_{(2,41)}=9.89\right.$, $p<0.001]$, and Connections Complex $\left[F_{(2,41)}=6.24, p<0.001\right]$, but not Connections Complex after controlling for Connections Simple 

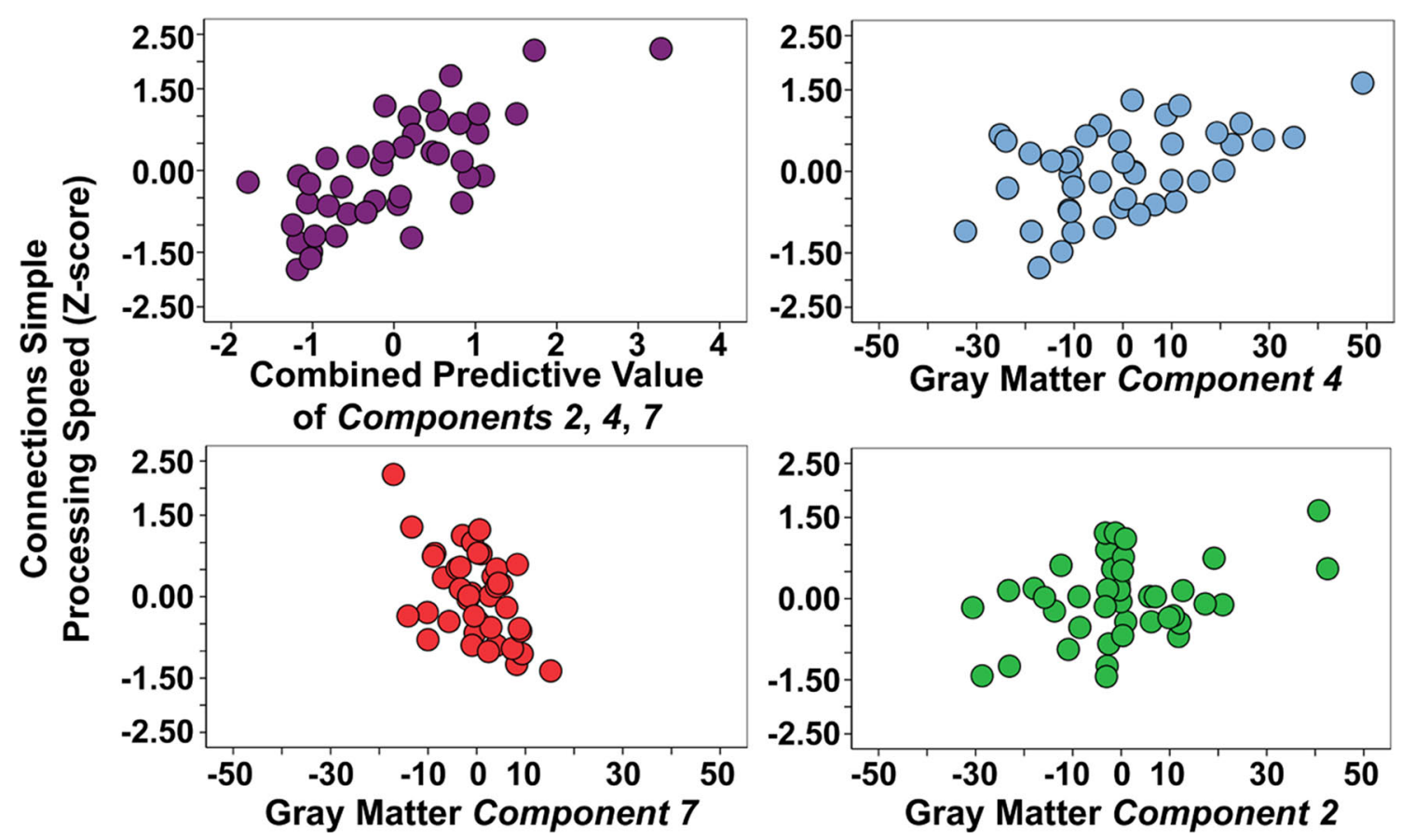

FIGURE 3 | Combined and unique gray matter components 2, 4, and $\mathbf{7}$ associations with Connections Simple processing speed. Note that gray matter component 2 is driven in part by two young subjects with elevated gray matter (positive values), which is represented by the relatively lower correlation estimates with Connections Simple presented in Table 3.

variance $\left[F_{(2,41)}=0.14, \mathrm{~ns}\right]$. Figures 6B,C demonstrates that perceptual and motor processing speed was particularly slow in people who had exhibited WMH posterolateral to the atrium of the lateral ventricle, anterior to the horn of the lateral ventricle and just superior to the body of the lateral ventricle in the corona radiata. People with the most pronounced WMH also demonstrated the most ventricle deformation during normalization (Figure 6C).

We also examined the extent to which the frequency of $\mathrm{WMH}$ was related to the gray matter components. There was a significant difference only for component $7\left[F_{(2,41)}=8.38, p<0.001\right]$. The gray matter volume estimate was increased in component 7 and decreased in component $7 a$ for subjects with the most WMH. For example, subjects with pronounced WMH had increased component 7 gray matter estimates in the white matter region anterior to the lateral ventricle $\left[F_{(2,41)}=13.89, p<0.001\right.$; region shown in Figure 2, 7] and decreased gray matter in ACC $\left[F_{(2,41)}=4.60, p<0.05\right.$; region shown in Figure 2, 7a].

Given the strong relation between WMH and gray matter component 7 , hierarchical multiple regression analysis was performed to determine the extent to which WMH explained the association between component 7 and processing speed. A regression nearly identical to the one described above was performed, with the exception that dummy variables representing no $\mathrm{WMH}$ and moderate to pronounced WMH were entered in the second level of the regression and redundant gray matter component 3 was not included. Table 4 shows that there was not a significant $R^{2}$ change with the addition of the WMH data. However, the unique predictive strength of component 7 was substantially reduced with the addition of the WMH dummy variables. The component 7 Std. Beta in Level 1 was
$-0.43, p<0.0005$ and with the inclusion of the WMH dummy variables in Level 2 the component 7 Std. Beta was $-0.30, p<0.05$. Components 4 and 7 were not unique predictors of processing speed when age was entered in the regression, again demonstrating that the association between these components and processing speed is age-dependent.

\section{DISCUSSION}

Age-related changes in processing speed have been attributed to structural declines in prefrontal cortex (Kennedy and Raz, 2009; Bartzokis et al., in press). We observed that a putative frontal network composed of ACC and dorsolateral prefrontal cortex regions predicted age-related changes in processing speed. This association appears to be driven by cerebral small vessel disease as the people with the most WMH exhibited the lowest gray matter volume in this frontal network and had the slowest processing speed. A putative cerebellar network also uniquely predicted processing speed, but was not related to cerebral small vessel disease, thereby demonstrating that age-related changes in at least two different networks contribute to age-related changes in processing speed.

\section{METHODOLOGICAL CONSIDERATIONS}

The age-related changes in processing speed that were observed in this study were most prominent for speeded perceptual and motor tasks with limited executive function demands. We excluded people with evidence of mild cognitive impairment in this study, which may have limited age-related changes in functions such as working memory. Therefore, the generalizability of our results may be 


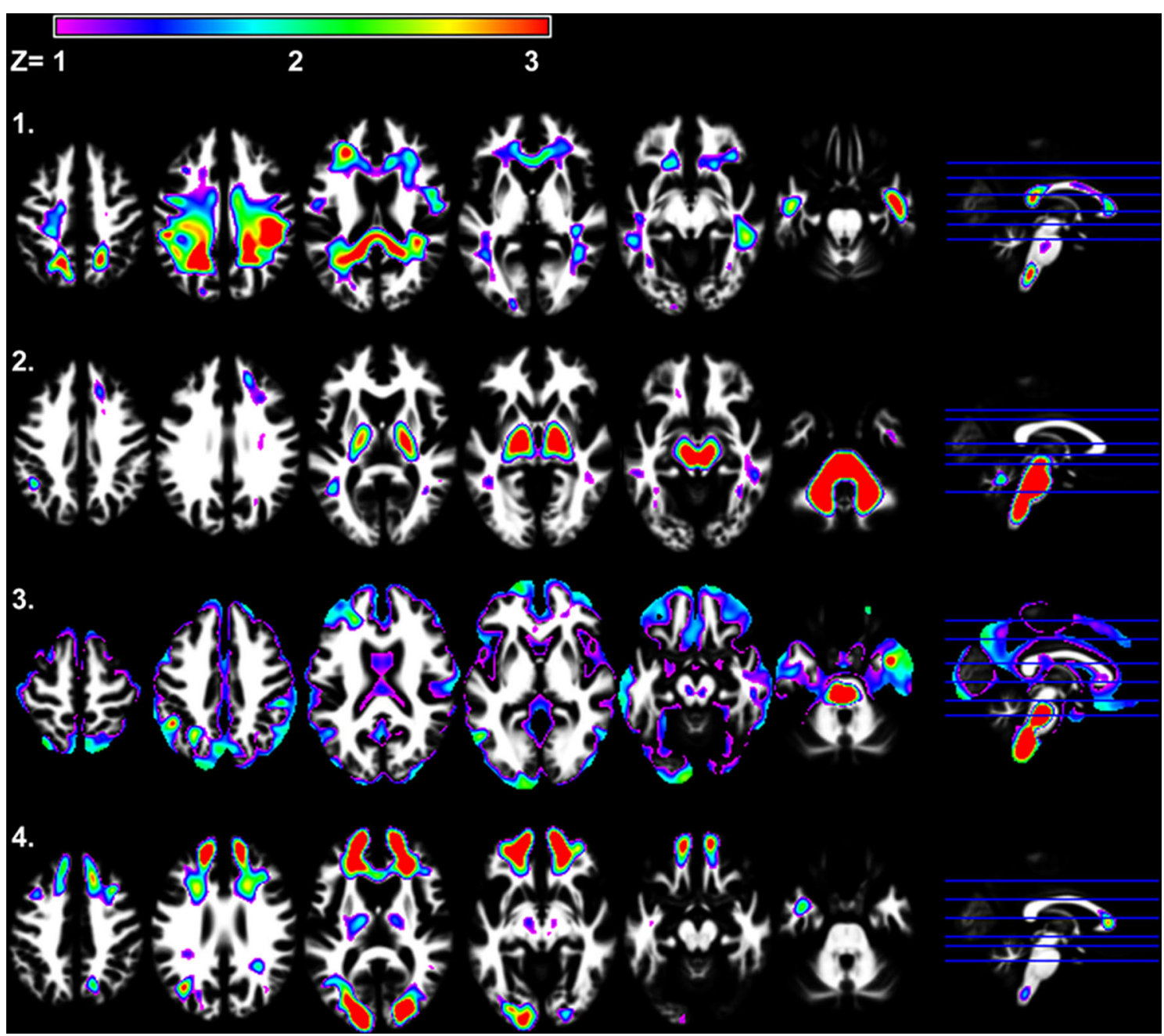

FIGURE 4 | SBM results demonstrating the four independent white matter components that were identified. White matter components 2 and 4 were significantly related to age $\left[r_{(41)}=-0.49, p<0.001\right.$ and $r_{(41)}=-0.32, p<0.05$, respectively]. Only white matter component 2 was significantly related to Connections Simple processing speed $\left[r_{(41)}=0.44, p<0.005\right]$

limited to relatively healthy older adults. In addition, there may be additional demographic factors that affect age-related changes in processing speed that we were not able to examine in our sample of 42 adults. For example, a larger number of older adults may have provided the opportunity to observe gender effects on vascular health and cognition (Raz et al., 2009). However, the results of this study are consistent with previous work demonstrating age-related changes in perceptual speed measured with the Connections Simple test, but not in working memory and response inhibition functions that are measured by the Connections Complex test (Salthouse, 2000). In addition, our study included subjects from across the adult lifespan and was not a study of changes in processing speed that occur in the oldest adults. Associations between brain morphology and processing speed across the adult lifespan were examined in this study because people exhibit changes in processing speed by the $4^{\text {th }}$ decade of life (Salthouse, 2000). Indeed, we observed evidence of cross-sectional perceptual and motor processing speed decline by 40 years of age.
We were able to identify unique associations between gray matter variation and processing speed using SBM with images that were carefully co-registered using the DARTEL procedure. These methods enabled us to disentangle and remove anatomical variation that could have otherwise obscured associations with processing speed. For example, we were able to determine that associations between gray matter and processing speed were unique to specific patterns of gray matter atrophy and not dependent on global changes in volume. While the DARTEL procedure appeared to successfully address the challenge of normalizing the images of older adults exhibiting large ventricles, there were white matter regions that were classified as gray matter (Figure 2 ). This occurred in regions where there was WMH evidence of cerebral small vessel disease (Figure 6; Kennedy and Raz, 2009). By using SBM, we were able to identify this WMH-related segmentation error and demonstrated that these $\mathrm{WMH}$ effects were relatively independent predictors of processing speech compared to other brain regions exhibiting associations with processing speed. 


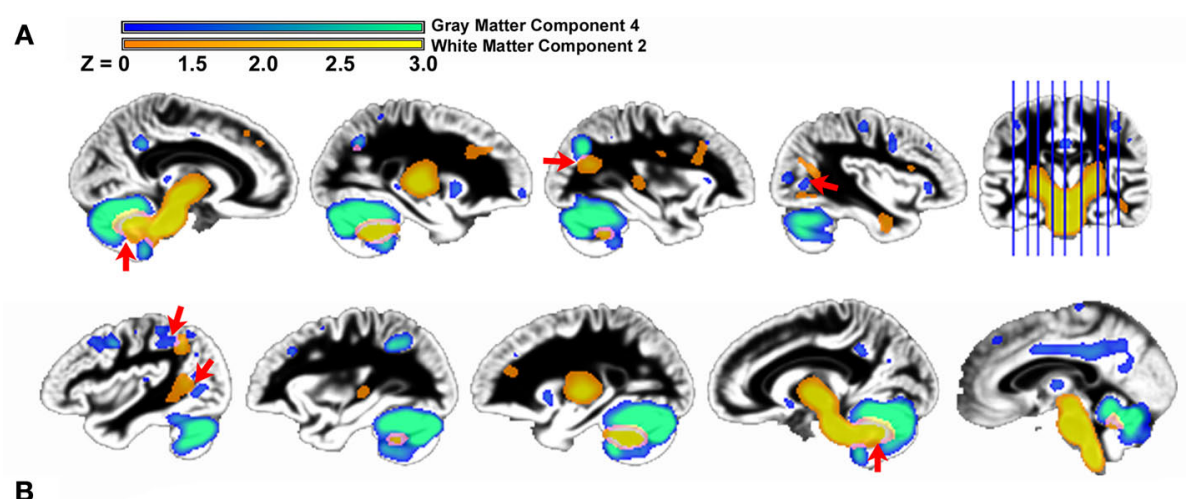

B

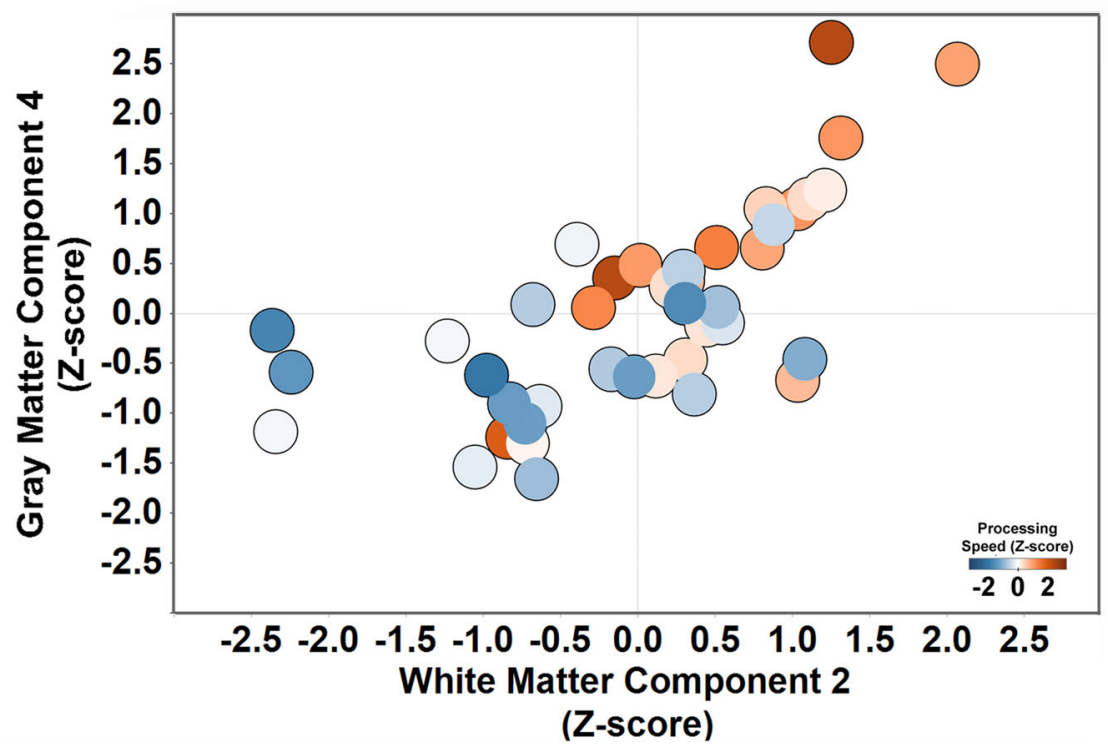

FIGURE 5 | Structural covariance between cerebellar gray matter and brainstem white matter. (A) Gray matter component 4 is displayed with white matter component 2 to demonstrate the space correspondence between the components (left hemisphere: top row; right hemisphere: bottom row). Note that the white matter component abuts regions of the gray matter component, including in regions throughout the cerebellum, parietal cortex, and parietal-occipital-temporal cortex (red arrows). (B) Individual variation in the volume estimates for gray matter component 4 and white matter component 2 are significantly correlated $\left[r_{(41)}=.68, p<0.001\right]$. People with elevated cerebellar gray matter had elevated white matter within regions that project to the cerebellum. The circles are shaded to indicate that both components predict performance on the Connections Simple processing speed measure.
The SBM procedure also identified patterns of structural covariance that are consistent with spatial patterns of functionally correlated brain regions. There was substantial spatial overlap between component 1 and the regions of the "default mode network" (Raichle et al., 2001; Greicius et al., 2003, 2009; Beckmann et al., 2005; Damoiseaux et al., 2006; Calhoun et al., 2008; Skudlarski et al., 2008), which was strongly associated with total gray matter volume. We also observed patterns of visual cortex (Beckmann et al., 2005; De Luca et al., 2006), cerebellar (Beckmann et al., 2005; Habas et al., 2009), anterior insula and parietal cortex (Beckmann et al., 2005; Damoiseaux et al., 2006), and bilateral temporal and prefrontal cortex (Beckmann et al., 2005; Habas et al., 2009) that have been related to functional networks. These observations are generally consistent with evidence for substantial overlap between spatial patterns of structural networks and low frequency fMRI functional data (Honey et al., 2007). Common patterns of spatial variance could reflect underlying connections (e.g., gray matter component 4 and white matter component 2), regions that vary together in the amount of neuropil and vascular support (e.g., gray matter component 1 ; note too that regions of the default mode network have been shown to be interconnected; Greicius et al., 2009), or are anatomically unconnected and driven by effects of cerebral small vessel disease that are in consistent locations across people (e.g., gray matter component 7).

\section{CEREBRAL SMALL VESSEL DISEASE}

One goal of this study was to determine the extent to which cerebral small vessel disease was related to structural changes within specific neural systems and slowed processing speed. Cerebral small vessel disease, as defined by the presence of $\mathrm{WMH}$, was related to perceptual and motor processing speed and gray matter component 7 . The association between processing speed and $\mathrm{WMH}$ is consistently reported in the literature (Gunning-Dixon and Raz, 2000) and robust in our sample given that all three WMH measures used in this study (qualitative assessment of WMH load, regional likelihood of WHM, and voxel-wise WMH mapping) were related to processing speed. 


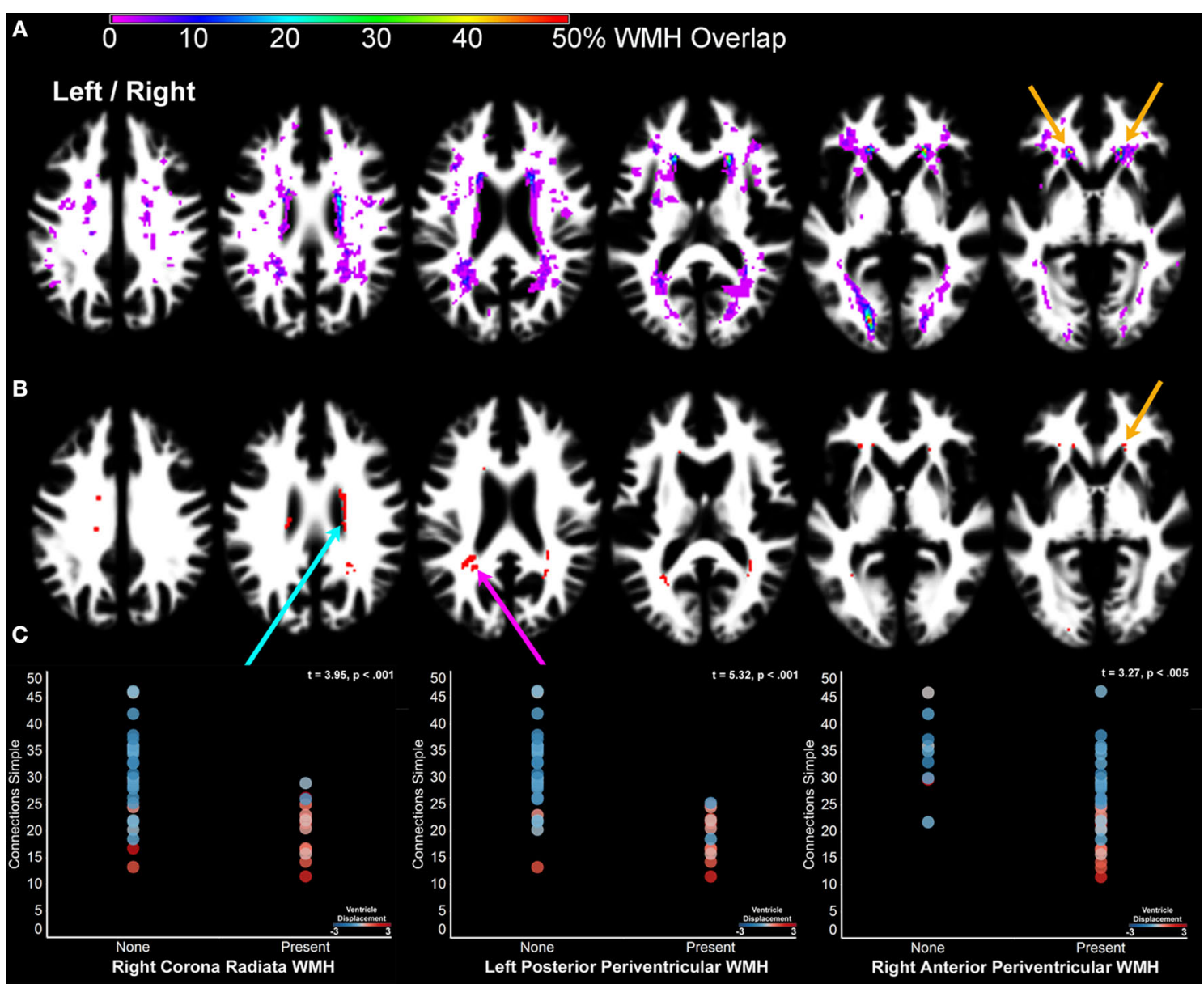

FIGURE 6 |The impact of cerebral small vessel disease on processing speed. (A) A probability map of WMH. WMH were observed with the highest probability in periventricular regions, as well as the optic radiations. The orange arrows indicate the location of WMH where white matter was segmented as gray matter and identified as part of component 7 (Figure $\mathbf{2}$ - orange arrow). (B) Nonparametric lesion-mapping results demonstrating locations of WMH most consistently associated with slow processing speed. (C) The presence of a WMH anywhere within the regions presented in (B) was determined for each subject in order to examine the impact of $\mathrm{WMH}$ in these regions on processing speed (1\% FDR = Z score threshold of 2.33). The scatter plots compare Connections Simple performance for people with and without WMH in the right corona radiata (blue arrow), left posterior periventricular white matter (pink arrow), and right anterior periventricular white matter (orange arrow) regions. The circles are shaded to indicate the degree to which each subject's lateral ventricles had to be displaced during normalization (Figure 1A). Note that the people with WMH had images requiring the most displacement during normalization [WMH classification comparison with ventricle displacement: $\left.F_{(2,41)}=8.95, p<.001\right]$. Left/right refers to left and right hemisphere.
WMH are often diffusely observed throughout the white matter but there are periventricular regions that are consistently affected in people (Figure 6A). These effects were identified in component 7 where there were regions that had to undergo significant deformation during normalization and where $\mathrm{WMH}$ were observed. For example, Figure 2 (orange arrows) indicates that anterior periventricular white matter regions were included in the gray matter component 7 where there was a relatively high probability of $\mathrm{WMH}$ (Figure 6A - orange arrows).

Cerebral small vessel disease appears to have cascading effects on brain morphology that include declines in frontal white matter, decreased $N$-acetylaspartate, a widening of sulcal width, and as a result slowed processing speed (Kochunov et al., 2010). In this study, people with WMH had less gray matter in ACC and dorsolateral prefrontal cortex, as well as slowed perceptual and motor processing speed. Atrophied gray matter within dorsolateral prefrontal cortex regions has been shown to exhibit reduced activity during working memory tasks in older adults with WMH (Nordahl et al., 2006). While the results of this study are consistent with the hypothesis that cerebral small vessel disease limits the role of ACC and dorsolateral prefrontal cortex to guide performance during cognitive tasks, there is a possibility that other etiological factors mediate the relation between age-related changes in prefrontal cortex and processing speed given the correlational design of this adult lifespan study.

\section{AGE-RELATED CHANGES IN PERCEPTUAL AND MOTOR VERSUS EXECUTIVE PROCESSING SPEED}

We expected to observe an age-related dissociation where a sensory and/or motor component would relate to perceptual and motor processing speed, while a frontal component would relate specifically to executive processing speed. Instead, the cerebellar and frontal components uniquely predicted perceptual and motor processing speed, while a component involving the anterior insula 
Table 4 | Hierarchical regression model demonstrating that WMH shares significant variance with gray matter component 7 in the prediction of Connections Simple processing speed.

\begin{tabular}{llrrl}
\hline Model & Variables & Std. beta & \multicolumn{1}{l}{$\boldsymbol{t}$} & Sig. \\
\hline$F=14.29$ & Component 2 & 0.26 & 2.27 & 0.0290 \\
$p<0.00001$ & Component 4 & 0.39 & 3.46 & 0.0014 \\
$R=0.73$ & Component 7 & $-\mathbf{0 . 4 3}$ & $-\mathbf{3 . 8 0}$ & $\mathbf{0 . 0 0 0 5}$ \\
$F=9.55$ & Component 2 & 0.24 & 2.01 & 0.0514 \\
$p<0.00001$ & Component 4 & 0.34 & 2.90 & 0.0062 \\
$R=0.76$ & Component 7 & $-\mathbf{0 . 3 0}$ & $-\mathbf{2 . 3 1}$ & $\mathbf{0 . 0 2 7 0}$ \\
$R^{2}=0.04, \mathrm{~ns}$ & WMH pronounced & -0.14 & -1.04 & $\mathrm{~ns}$ \\
& WMH none & 0.15 & 1.10 & $\mathrm{~ns}$ \\
$F=9.70$ & Component 2 & 0.27 & 2.36 & 0.0243 \\
$p<0.00001$ & Component 4 & 0.12 & 0.82 & $\mathrm{~ns}$ \\
$R=0.79$ & Component 7 & -0.19 & -1.37 & $\mathrm{~ns}$ \\
$R^{2}=0.05, p<0.05$ & WMH pronounced & -0.09 & -0.67 & $\mathrm{~ns}$ \\
& WMH none & -0.01 & -0.04 & $\mathrm{~ns}$ \\
& Age & -0.44 & -2.25 & 0.0310
\end{tabular}

The significant values are indicated by bold.

and caudate (component 6) predicted executive processing speed but this relation was not dependent on age. Perhaps age-dependent changes in executive processing speed would have been observed had the sample included people over 80 years or who exhibited evidence of mild cognitive impairment.

One assumption in defining the cognitive demands of the Connections Simple task is that it involves limited executive demands (e.g., working memory or response inhibition). The task still requires, however, decision making and planning. In that context, the ability to stay focused on the task and prepare a response may be dependent on frontal networks that are affected by cerebral small vessel disease. This may explain the age-dependent association between performance on the Connections Simple and the Numbers Reversed working memory tasks in this study and previous findings linking cerebral small vessel disease to changes in working memory (Nordahl et al., 2006). Alternatively, age-related variance in working memory could simply be accounted for by perceptual and motor contributions to processing speed in this sample.

\section{THE CEREBELLUM AND SLOWED PROCESSING SPEED}

One important and novel finding in this study is that perceptual and motor processing speed performance was predicted by a gray matter component largely represented by the cerebellum. The role of the cerebellum in rapid information processing and modifying motor function in response to sensory and perceptual feedback (Ivry, 1997) is broadly consistent with the behavioral demands of perceptual and motor processing speed tasks. Indeed, processing speed has been related to the degree of task-related cerebellar activation in healthy adults (Genova et al., 2009), variation in cerebellar fractional anisotropy in young adults (Bohr et al., 2007), and cerebellar lesion patients (Archibald et al., 2004).

Cerebellar morphology has been associated with cognitive abilities (Eckert et al., 2003; Chen and Desmond, 2005; Desmond et al., 2005; Steinlin, 2007) and the cerebellum exhibits distinct patterns of structural and functional connectivity with motor, sensory, and attention-related cortical regions (Kelly and Strick, 2003; Habas et al., 2009; Krienen and Buckner, 2009) that would be critical for using perceptual information to optimize motor control. In this study, the cerebellar regions contributing the most variation to component 4 were hemispheric crus 1 and crus 2 regions that have been reported to exhibit functional connectivity with parietal cortex (Habas et al., 2009) and dorsolateral prefrontal cortex (Habas et al., 2009; Krienen and Buckner, 2009). These cerebellar and frontal/parietal regions also exhibit elevated and correlated activity when subjects must integrate spatial orientation and velocity information to predict where an object will appear (O'Reilly et al., 2008), a task that is somewhat analogous to the processing speed tasks used in this study whereby subjects must anticipate and orient to the location of the next object to perform a rapid yet controlled hand and arm movement. Interestingly, cerebellar component 4 also included a left intraparietal sulcus region, further suggesting that we observed structural networks that overlap spatially with functional networks. Together these findings guide the prediction that aging disrupts the coordination of cerebellar and frontal/parietal regions, with slowed motor and perceptual processing speed as a consequence.

Age-related declines in cerebellar gray matter volume have been reported previously (Sullivan et al., 2000; Good et al., 2001; Lee et al., 2005; Raz et al., 2005; Harris et al., 2009). However, the significance of these changes has not been clear. Disrupted coordination between cerebellar and frontal/parietal regions could explain, in part, why some older adults demonstrate slower rates of perceptual-motor learning (Rodrigue et al., 2005). Reduced cerebellar volume has been associated with age-related declines in procedural learning ( $R a z$ et al., 2000), an estimate of spatial intelligence (Lee et al., 2005), and a study on age-related changes in gait control has linked cerebellar morphology to impaired motor speed and balance (Rosano et al., 2007). In accordance with the findings from this study, WMH did not explain the relation between cerebellar hemisphere volumes and motor function reported by Rosano et al. (2007). While the etiological factors driving age-related changes in cerebellar morphology are yet to be determined, oxidative stress is one factor contributing to cerebellar aging in rodent models (Lee et al., 2000). Interestingly, a physically active lifestyle compared to a sedentary lifestyle in rodents is associated with reduced DNA oxidation (Cui et al., 2009). In humans, older adults who exercise may experience limited declines in cerebellar morphology (Colcombe et al., 2003), thereby limiting age-related declines in processing speed (Rikli and Edwards, 1991).

\section{CONCLUSION}

The results from this study demonstrate that age-related declines in processing speed stem from changes in at least two different networks: (1) a putative frontal network related to cerebral small vessel disease and (2) a putative cerebellar network that was not affected by cerebral small vessel disease. There remained unexplained variance in perceptual and motor processing speed suggesting that the full impact of cerebral small vessel disease may have been under-estimated and that additional factors that may further 
elucidate age-related changes in processing speed. For example, the white matter components identified in this study were not strong predictors of processing speed even though WMH had a relatively large impact on perceptual and motor processing speed. Direct measures of changes within major fiber bundles may be more sensitive to age-related changes in processing speed (Kennedy and Raz, 2009). In addition, the changes in GABAergic function within sensory cortex observed in rodents (Caspary et al., 2008) may decrease the quality of perceptual representations and as a consequence slow information processing. Nonetheless, the results of this study highlight targets for limiting the effects of age on perceptual and motor processing speed. Interventions designed to improve perceptual-motor integration through cerebellar plasticity may limit the global declines in cognition that are often attributed to declines in processing speed.

\section{ACKNOWLEDGMENTS}

We would like to thank the participants of this study. This work was supported by the National Institute on Deafness and other Communication Disorders (P50 DC00422) and was conducted in a facility constructed with support from Research Facilities Improvement Program (C06 RR14516) from the National Center for Research Resources, while Mark A. Eckert was an American Federation for Aging Research Grant recipient.

\section{REFERENCES}

Archibald, C. J., Wei, X., Scott, J. N., Wallace, C. J., Zhang, Y., Metz, L. M., and Mitchell, J. R. (2004). Posterior fossa lesion volume and slowed information processing in multiple sclerosis. Brain 127, 1526-1534.

Ashburner, J. (2007). A fast diffeomorphic image registration algorithm. Neuroimage 38, 95-113.

Ashburner, J., and Friston, K. J. (2005). Unified segmentation. Neuroimage $26,839-851$.

Au, R., Massaro, J. M., Wolf, P. A., Young, M. E., Beiser, A., Seshadri, S., D'Agostino, R. B., and DeCarli, C. (2006). Association of white matter hyperintensity volume with decreased cognitive functioning: the Framingham Heart Study. Arch. Neurol. 63, 246-250.

Bartzokis, G, Lu, P. H., Tingus, K., Mendez, M. F., Richard, A., Peters, D. G., Oluwadara, B., Barrall, K. A., Finn, J. P., Villablanca, P., Thompson, P. M., and Mintz, J. (in press). Lifespan trajectory of myelin integrity and maximum motor speed. Neurobiol. Aging doi: 10.1016 .

Beckmann, C. F., DeLuca, M., Devlin, J.T., and Smith, S. M. (2005). Investigations into resting-state connectivity using independent component analysis. Philos. Trans. R. Soc. Lond., B, Biol. Sci. 360, 1001-1013.

Bell, A. J., and Sejnowski, T. J. (1995). An information-maximization approach to blind separation and blind deconvolution. Neural. Comput. 7, 1129-1159.

Bohr,S., Gullmar,D., Knab,R., Reichenbach, J. R., Witte, O. W., and Haueisen, J. (2007). Fractional anisotropy correlates with auditory simple reaction time performance. Brain Res. 1186, 194-202.

Bullmore, E., and Sporns, O. (2009). Complex brain networks: graph theoretical analysis of structural and functional systems. Nat. Rev. Neurosci. 10, 186-198.

Calhoun, V. D., Kiehl, K. A., and Pearlson, G.D. (2008).Modulation of temporally coherent brain networks estimated using ICA at rest and during cognitive tasks. Hum. Brain Mapp. 29, 828-838.

Caspary, D. M., Ling, L., Turner, J. G., and Hughes, L. F. (2008). Inhibitory neurotransmission, plasticity and aging in the mammalian central auditory system. J. Exp. Biol. 211, 1781-1791.

Charlton, R. A., Barrick, T. R., McIntyre, D. J., Shen, Y., O'Sullivan, M., Howe, F. A., Clark, C. A., Morris, R. G., and Markus, H. S. (2006). White matter damage on diffusion tensor imaging correlates with age-related cognitive decline. Neurology 66, 217-222.

Chen, S. H., and Desmond, J. E. (2005). Temporal dynamics of cerebro-cerebellar network recruitment during a cognitive task. Neuropsychologia 43, 1227-1237.

Coffey, C. E., Ratcliff, G., Saxton, J. A., Bryan, R. N., Fried, L. P., and Lucke, J.F. (2001). Cognitive correlates of human brain aging: a quantitative magnetic resonance imaging investigation. $J$. Neuropsychiatry Clin. Neurosci. 13, 471-485.

Colcombe, S. J., Erickson, K. I., Raz, N., Webb, A. G., Cohen, N. J., McAuley, E., and Kramer, A. F. (2003). Aerobic fitness reduces brain tissue loss in aging humans. J. Gerontol. A Biol. Sci. Med. Sci. 58, 176-180.

Cui, L., Hofer, T., Rani, A., Leeuwenburgh, C., and Foster, T. C. (2009). Comparison of lifelong and late life exercise on oxidative stress in the cerebellum. Neurobiol. Aging 30, 903-909.

Damoiseaux, J. S., Rombouts, S. A., Barkhof, F., Scheltens, P., Stam, C. J., Smith, S. M., and Beckmann, C. F. (2006). Consistent resting-state networks across healthy subjects. Proc. Natl. Acad. Sci. U.S.A. 103, 13848-13853.

De Luca, M., Beckmann, C. F., De Stefano, N., Matthews, P. M., and Smith, S. M. (2006). fMRI resting state networks define distinct modes of long-distance interactions in the human brain Neuroimage 29, 1359-1367.

Desmond, J. E., Chen, S. H., and Shieh, P. B. (2005). Cerebellar transcranial magnetic stimulation impairs verbal working memory. Ann. Neurol. 58, 553-560.

Eckert, M. A., Leonard, C. M., Richards, T. L., Aylward, E. H., Thomson, J., and Berninger, V. W. (2003). Anatomical correlates of dyslexia: frontal and cerebellar findings. Brain 126, 482-494.

Genova, H. M., Hillary, F. G., Wylie, G., Rypma, B., and Deluca, J. (2009). Examination of processing speed deficits in multiple sclerosis using functional magnetic resonance imaging. $J$. Int. Neuropsychol. Soc. 15, 383-393.

Good, C. D., Johnsrude, I. S., Ashburner, J., Henson, R. N., Friston, K. J., and Frackowiak, R. S. (2001). A voxelbased morphometric study of ageing in 465 normal adult human brains. Neuroimage 14, 21-36.

Greicius, M. D., Krasnow, B., Reiss, A. L., and Menon, V. (2003). Functional connectivity in the resting brain: a network analysis of the default mode hypothesis. Proc. Natl. Acad. Sci. U.S.A. 100, 253-258.

Greicius, M. D., Supekar, K., Menon, V., and Dougherty, R. F. (2009). Restingstate functional connectivity reflects structural connectivity in the default mode network. Cereb. Cortex 19, 72-78.

Gunning-Dixon, F.M., and Raz, N. (2000). The cognitive correlates of white matter abnormalities in normal aging: a quantitative review. Neuropsychology 14, 224-232.

Habas, C., Kamdar, N., Nguyen, D., Prater, K., Beckmann, C. F., Menon, V., and Greicius, M. D. (2009). Distinct cerebellar contributions to intrinsic connectivity networks. J. Neurosci. 29, 8586-8594.

Harris, K. C., Dubno, J. R., Keren, N. I., Ahlstrom, J. B., and Eckert, M. A. (2009). Speech recognition in younger and older adults: a dependency on low-level auditory cortex. J. Neurosci. 29, 6078-6087.

He, Y., Chen, Z., and Evans, A. (2008). Structural insights into aberrant topological patterns of large-scale cortical networks in Alzheimer's disease. J. Neurosci. 28, 4756-4766.

He, Y., Chen, Z. J., and Evans, A. C. (2007). Small-world anatomical networks in the human brain revealed by cortical thickness from MRI. Cereb. Cortex 17 2407-2419.

He, Y., Dagher, A., Chen, Z., Charil, A., Zijdenbos, A., Worsley, K., and Evans, A. (2009). Impaired small-world efficiency in structural cortical networks in multiple sclerosis associated with white matter lesion load. Brain 132 3366-3379.

Honey, C. J., Kotter, R., Breakspear, M., and Sporns, O. (2007). Network structure of cerebral cortex shapes functional connectivity on multiple time scales. Proc. Natl. Acad. Sci. U.S.A. 104, 10240-10245.

Ivry, R. (1997). Cerebellar timing systems. Int. Rev. Neurobiol. 41, 555-573.

Kelly, R. M., and Strick, P. L. (2003). Cerebellar loops with motor cortex and prefrontal cortex of a nonhuman primate. J. Neurosci. 23, 8432-8444.

Kennedy, K. M., and Raz, N. (2009). Aging white matter and cognition: differential effects of regional variations in diffusion properties on memory, executive functions, and speed. Neuropsychologia 47, 916-927.

Kochunov, P., Coyle, T., Lancaster, J., Robin, D., Hardies, J., Kochunov, V., Bartzonkis, G., Stanley, J., Royall, D., Schlosser, A., Null, M., and Fox, P. (2010). Processing speed is correlated with cerebral health markers in the frontal lobes as quantified by neuro-imaging. Neuroimage 49 , 1190-1199.

Krienen, F. M., and Buckner, R. L. (2009). Segregated fronto-cerebellar circuits revealed by intrinsic functional connectivity. Cereb. Cortex 19, 2485-2497.

Lee, C. K., Weindruch, R., and Prolla, T. A. (2000). Gene-expression profile of 
the ageing brain in mice. Nat. Genet. 25, 294-297.

Lee, J. Y., Lyoo, I. K., Kim, S. U., Jang, H. S., Lee, D. W., Jeon, H. J., Park, S. C., and Cho, M. J. (2005). Intellect declines in healthy elderly subjects and cerebellum. Psychiatry Clin. Neurosci. 59, 45-51.

Li, Y. O., Adali, T., and Calhoun, V. D. (2007). Estimating the number of independent components for functional magnetic resonance imaging data. Hum. Brain Mapp. 28, 1251-1266.

Miller, E. M. (1994). Intelligence and brain myelination: a hypothesis. Pers. Individ. Dif. 17, 803-832.

Morris, J.C., and McManus, D. Q. (1991). The neurology of aging: normal versus pathologic change. Geriatrics 46 , $47-48,51-44$.

Nordahl, C.W., Ranganath, C., Yonelinas, A. P., Decarli, C., Fletcher, E., and Jagust, W. J. (2006). White matter changes compromise prefrontal cortex function in healthy elderly individuals. J. Cogn. Neurosci. 18, 418-429.

O'Reilly, J.X., Mesulam, M.M., and Nobre, A. C. (2008). The cerebellum predicts the timing of perceptual events. $J$. Neurosci. 28, 2252-2260.

Prins, N. D., van Dijk, E. J., den Heijer, T., Vermeer, S. E., Jolles, J., Koudstaal, P. J., Hofman, A., and Breteler, M. M. (2005). Cerebral small-vessel disease and decline in information processing speed, executive function and memory. Brain 128, 2034-2041.

Raichle, M.E., MacLeod, A. M., Snyder,A. Z., Powers, W. J., Gusnard, D. A., and Shulman, G.L. (2001). A default mode of brain function. Proc. Natl. Acad. Sci. U.S.A. 98, 676-682.

Raz, N., Lindenberger, U., Rodrigue, K.M., Kennedy, K.M., Head, D., Williamson, A., Dahle, C., Gerstorf, D., and Acker, J.D. (2005). Regional brain changes in aging healthy adults: general trends, individual differences and modifiers. Cereb. Cortex 15, 1676-1689.

Raz, N., Rodrigue, K. M., Kennedy, K., and Land, S. (2009). Genetic and vascular modifiers of age-sensitive cognitive skills. Effects of COMT, BDNF, ApoE, and hypertension. Neuropsychology 23 , 105-116.

Raz, N., Williamson, A., Gunning-Dixon, F., Head, D., and Acker, J. D. (2000). Neuroanatomical and cognitive correlates of adult age differences in acquisition of a perceptual-motor skill. Microsc. Res. Tech. 51, 85-93.

Reitan, R. M. (1992). Trail Making Test: Manual for Administration and Coring. Tucson, AZ, Reitan Neuropsychology Laboratory.

Rikli, R. E., and Edwards, D. J. (1991). Effects of a three-year exercise program on motor function and cognitive processing speed in older women. Res. Q. Exerc. Sport 62, 61-67.

Rodrigue, K. M., Kennedy, K. M., and Raz, N. (2005). Aging and longitudinal change in perceptual-motor skill acquisition in healthy adults. J. Gerontol. B. Psychol. Sci. Soc. Sci. 60, P174-P181.

Rorden, C., Karnath, H. O., and Bonilha, L. (2007). Improving lesion-symptom mapping. J. Cogn. Neurosci. 19, 1081-1088.

Rosano, C., Aizenstein, H. J., Studenski, S., and Newman, A. B. (2007). A regionsof-interest volumetric analysis of mobility limitations in communitydwelling older adults. J. Gerontol. A Biol. Sci. Med. Sci. 62, 1048-1055.

Salthouse, T. A. (1992). Mechanisms of Age-Cognition Relations in Adulthood. Hillsdale, NJ, Lawrence Erlbaum.

Salthouse, T. A. (1996). The processingspeed theory of adult age differences in cognition. Psychol. Rev. 103, 403-428.

Salthouse, T. A. (2000). Aging and measures of processing speed. Biol. Psychol. $54,35-54$.

Salthouse, T.A. (2005). Relations between cognitive abilities and measures of executive functioning. Neuropsychology 19, 532-545.

Salthouse, T. A., and Ferrer-Caja, E. (2003). What needs to be explained to account for age-related effects on multiple cognitive variables? Psychol. Aging 18, 91-110.

Salthouse, T. A., Toth, J., Daniels, K., Parks, C., Pak, R., Wolbrette, M., and Hocking, K. J. (2000). Effects of aging on efficiency of task switching in a variant of the trail making test. Neuropsychology 14, 102-111.

Schiavone, F., Charlton, R. A., Barrick, T. R., Morris, R. G., and Markus, H. S. (2009). Imaging age-related cognitive decline: a comparison of diffusion tensor and magnetization transfer MRI.J. Magn. Reson. Imaging 29, 23-30.

Singer, T., Verhaeghen, P., Ghisletta, P., Lindenberger, U., and Baltes, P. B. (2003). The fate of cognition in very old age: six-year longitudinal findings in the Berlin Aging Study (BASE). Psychol. Aging 18, 318-331.

Skudlarski, P., Jagannathan, K., Calhoun, V.D., Hampson, M., Skudlarska, B. A. and Pearlson, G. (2008). Measuring brain connectivity: diffusion tensor imaging validates resting state temporal correlations. Neuroimage 43, 554-561.

Steinlin, M. (2007). The cerebellum in cognitive processes: supporting studies in children. Cerebellum 6, 237-241.

Sullivan, E. V., Deshmukh, A., Desmond, J. E., Lim, K. O., and Pfefferbaum, A. (2000). Cerebellar volume decline in normal aging, alcoholism, and Korsakoff's syndrome: relation to ataxia. Neuropsychology 14, 341-352.

Trebbe, O., Schwindt, W., Mohammadi, H., Wersching, H., and Deppe, M. (2009). Automated segmentation and spatial registration of white matter lesions in MR FLAIR images. Neuroimage 47(Suppl. 1), S90.

van den Heuvel, D. M., ten Dam, V. H., de Craen, A. J., Admiraal-Behloul, F., Olofsen, H., Bollen, E. L., Jolles, J., Murray, H. M., Blauw, G. J., Westendorp, R. G., and van Buchem, M. A. (2006). Increase in periventricular white matter hyperintensities parallels decline in mental processing speed in a non-demented elderly population.J. Neurol. Neurosurg. Psychiatr. 77, 149-153.

van Dijk, E. J., Prins, N. D., Vrooman, H. A., Hofman, A., Koudstaal, P. J., and Breteler, M. M. (2008). Progression of cerebral small vessel disease in relation to risk factors and cognitive consequences: Rotterdam Scan Study. Stroke 39, 2712-2719.

Vernooij, M. W., de Groot, M., van der Lugt, A., Ikram, M. A., Krestin, G. P., Hofman, A., Niessen, W. J., and Breteler, M. M. (2008). White matter atrophy and lesion formation explain the loss of structural integrity of white matter in aging. Neuroimage 43, 470-477.

Welford, A. T. (1981). Signal, noise, performance, and age. Hum. Factors 23, 97-109.

Woodcock, R. W., McGrew, K. S., and Mather, N. (2001). WoodcockJohnson Tests of Achievement (WJ III-R). Itasca, IL, Riverside

Xu, L., Pearlson, G., and Calhoun, V. D. (2009a). Joint source based morphometry identifies linked gray and white matter group differences. Neuroimage 44, 777-789.

Xu, L., Groth, K., Pearlson, G., Schretlen, D., and Calhoun, V. (2009b). Source based morphometry: the use of independent component analysis to identify gray matter differences with application to schizophrenia. Hum. Brain Mapp. 30, 711-724.

Conflict of Interest Statement: The authors declare that the research was conducted in the absence of any commercial or financial relationships that could be construed as a potential conflict of interest.

Received: 13 November 2009; paper pending published: 03 January 2010; accepted: 24 January 2010; published online: 08 March 2010.

Citation: Eckert MA, Keren NI, RobertsDR, Calhoun VD and Harris KC (2010) Agerelated changes in processing speed: unique contributions of cerebellar and prefrontal cortex. Front. Hum. Neurosci. 4:10. doi: 10.3389/neuro.09.010.2010

Copyright (๑) 2010 Eckert, Keren, Roberts, Calhoun and Harris. This is an open-access article subject to an exclusive license agreement between the authors and the Frontiers Research Foundation, which permits unrestricted use, distribution, and reproduction in any medium, provided the original authors and source are credited. 